\title{
Remoción de cromo hexavalente en aguas contaminadas utilizando cáscara de plátano (Musa paradisiaca) como adsorbente
}

Lic. Maybis López Hernández Especialista en análisis de laboratorio. Laboratorio de Biotecnología UNAN-Managua https://orcid.org/0000-0002-4523-3129 mlopezh@unan.edu.ni

Dra. Martha Lacayo Romero Docente titular, Laboratorio de Biotecnología UNAN-Managua https://orcid.org/0000-0002-6918-7796 mlacayor@unan.edu.ni

Palabras clave: cromo hexavalente, biomasa, modelos de isotermas, remoción, adsorción

\section{RESUMEN}

1 objetivo de esta investigación fue remover el cromo hexavalente de aguas - contaminadas utilizando cáscara de plátano como adsorbente. El estudio se realizó en dos etapas: en la primera etapa, se trabajó con $1 \mathrm{~g}$ de la biomasa a un tamaño de partícula de aproximadamente $600 \mu \mathrm{m}, 15$ minutos de contacto, $20 \mathrm{mg} \cdot \mathrm{l}^{-1}$ de cromo hexavalente y a pH 3, 3,5, 5 y 7 . En la segunda etapa, se trabajó con las mismas condiciones de masa y tiempo a pH 3 y 3,5 aumentando la concentración del metal de 20 a 40 y 80 mg.l $\mathrm{l}^{-1}$. Los resultados obtenidos muestran que al aumentar el $\mathrm{pH}$ disminuye el porcentaje de remoción y la capacidad de adsorción, no obstante, a pH 3 y 3,5 la biomasa remueve más del 92\% de cromo 
hexavalente y al aumentar la concentración aumenta la capacidad de adsorción de la cáscara de plátano. Los datos experimentales se ajustan más al modelo de Langmuir que al de Freundlich lo que muestra una adsorción en monocapa de una superficie homogénea. Además, el factor de separación $R_{L}$ se mantuvo entre 0,809 a 0,383 lo que indica una adsorción favorable del metal en la biomasa. La cáscara de plátano tiene gran potencial como adsorbente para la remoción de cromo hexavalente en aguas.

\section{INTRODUCCIÓN}

La contaminación del agua por metales pesados representa un problema ambiental importante debido a los efectos tóxicos que provocan a la salud humana. Las principales fuentes de contaminación del agua por metales pesados provienen de las aguas residuales industriales tales como metalizado, minería, curtiembres, pinturas, fabricación de radiadores, fundición, industrias de aleaciones, entre otros. (Al-Azzawi, M.N.A., Shartooh, S.M., Al-Hiyaly S.A.K., 2013). La descarga máxima permisible para cromo hexavalente hacia cuerpos receptores debe ser menor a 0,5 mg. l-1 $^{-1}$ gún el reglamento en el que se establecen las disposiciones para el vertido de aguas residuales decreto ejecutivo $N^{\circ}$. 21-2017. (Asamblea Nacional, 2017). El cromo existe en más de un estado de oxidación desde $\mathrm{Cr}$ (0) la forma metálica, hasta su forma hexavalente, $\mathrm{Cr}$ (VI). Especialmente, el cromo en su estado de oxidación +6 es considerado peligroso incluso en pequeñas concentraciones, mientras que $\mathrm{Cr}$ en estado de oxidación +3 es esencial para la salud en condiciones moderadas. (Guertin J., 2004)

El cromo hexavalente puede ocasionar efectos adversos a corto y a largo plazo, siendo el tracto respiratorio el principal órgano afectado después de la exposición. El cromo hexavalente puede ocasionar cáncer de pulmón, irritación o daños en la piel, ojos, nariz y garganta. Según la OSHA, el valor límite de exposición permisible de cromo hexavalente por día debe ser $5 \mu \mathrm{g} \cdot \mathrm{m}^{-3}$. (OSHA , 2009). La exposición por inhalación crónica al cromo hexavalente en humanos produce perforaciones y ulceraciones del tabique, bronquitis, disminución de la función pulmonar, neumonía, asma y picazón nasal. Los altos niveles de cromo hexavalente pueden producir efectos en el hígado, los riñones, sistema gastrointestinal e inmunitario. (EPA, 2000)

En las aguas residuales el cromo hexavalente se encuentra en solución como $\mathrm{CrO}_{4}{ }^{2}$, que puede ser removido por reducción, precipitación química, por adsorción y por intercambio iónico. El proceso más utilizado es la adición de un agente reductor que convierta el cromo hexavalente a cromo trivalente y posteriormente la precipitación con soluciones básicas $\mathrm{Cr}(\mathrm{OH})_{3}$. Actualmente, se ha analizado el uso de metodologías alternativas como la remoción y/o reducción del cromo hexavalente al cromo trivalente por bacterias, algas, levaduras y hongos. (Torres, L. , Cárdenas, J. F., Moctezuma, M. G., Martínez, V. M., Acosta, I., 2012) Los residuos agrícolas disponibles son una opción viable para la remediación de metales pesados ya que son considerados una fuente 
importante para la adsorción. En este contexto, la cáscara de plátano puede evaluarse como un adsorbente para la remoción de cromo hexavalente. (Díaz, M. R., Contreras, R., Guardiola, M. A., Mayo del Río, C., 2016)

La cáscara de plátano según estudio realizado por Kamsonlian, S., Suresh, S., Majumder, C.B., Chand, S., 2011 posee superficies irregulares y porosas, proteínas y polisacáridos dentro de su cubierta celular, algunos grupos funcionales $\mathrm{N}-\mathrm{H}$ y O-H, grupos carboxilos, anillos aromáticos $C=C$ y presencia de sílice que le brinda a la biomasa propiedades para su uso de manera fructífera como adsorbente en el tratamiento de aguas contaminadas. Kamsonlian S. et al. (2011)

El objetivo de esta investigación fue presentar una alternativa eficaz y de bajo costo para la remoción de cromo hexavalente en aguas contaminadas, mediante la utilización de cascara de plátano (Musa paradisiaca) como adsorbente para remover cromo hexavalente, tomando en consideración ciertos parámetros que influyen en el proceso de remoción tales como: pH de la solución, concentración del metal y tiempo de contacto. La capacidad máxima de adsorción de la biomasa se determinó mediante los modelos de isotermas de Langmuir y Freundlich.

\section{MATERIALES Y MÉTODOS}

\section{Preparación de la cáscara de plátano}

La cáscara de plátano fue obtenida del comedor Central de la Universidad Nacional Autónoma de Nicaragua, Managua (UNAN-Managua) y del comedor del Centro para la Investigación de Recursos Acuáticos (CIRA/UNAN-Managua) las cuales fueron recolectadas en sacos y llevadas al Laboratorio de Biotecnología donde fueron limpiadas con agua desionizada y cortadas en partes más pequeñas. Las cáscaras fueron puestas a secar a temperatura ambiente por 3 días, luego introducidas al horno marca Thermo Scientific a $60^{\circ} \mathrm{C}$ por 2 horas y finalmente trituradas en un molino manual casero, marca Victor para obtener un tamaño de partícula de aproximadamente $600 \mu \mathrm{m}$. La harina de cáscara de plátano fue almacenada en bolsas Ziploc dentro de un frasco hermético para su posterior uso.

\section{Fase experimental}

Para evaluar la capacidad de adsorción de la cáscara de plátano, se tomó en cuenta la influencia del pH en la remoción del metal y las concentraciones iniciales del cromo hexavalente. Los ensayos experimentales fueron realizados a escala de laboratorio por duplicado con el fin de evaluar la repetitividad del análisis. En la primera etapa, se trabajó con $\mathrm{pH}$ de 3, 3,5, 5 y 7 con un $1 \mathrm{~g}$ de cáscara de plátano por cada $100 \mathrm{ml}$ agua contaminada a una concentración de $20 \mathrm{mg} \cdot \mathrm{l}^{-1} \mathrm{de}$ cromo hexavalente durante 15 minutos en agitación constante de aproximadamente $600 \mathrm{rpm}$ en un plato de agitación marca Cimarec. En la segunda etapa, se trabajó con concentraciones de 20, 40, y 80 mg. $\mathrm{l}^{-1}$ de cromo hexavalente a pH 3 y 3,5 durante 15 minutos en agitación constante. 
En cada etapa, se tomaron alícuotas al 0, 2, 4, 6, 10 y 15 minutos de contacto con la cáscara de plátano, lo cuales fueron pasadas a través de un filtro Whatman $110 \mathrm{~mm}$.

La determinación del cromo hexavalente se realizó en un espectrofotómetro UV-vis marca Varian Cary 50 a una absorbancia de 540 nm, mediante el método colorimétrico de la 1,5 difenilcarbazida descrito en el 3500-Cr B Standard Methods. (APHA, AWWA, WEF, 2012). Se utilizó el software SigmaPlot 14.0 para la obtención de los gráficos.

\section{RESULTADOS Y DISCUSIÓN}

El porcentaje de remoción de los iones de cromo hexavalente y la cantidad de iones absorbidos en la cáscara de plátano (qe), fue calculado mediante las ecuaciones (1) y (2).

(1) $\%$ Remoción $=\frac{100(\mathrm{C} 0-\mathrm{Ceq})}{\mathrm{co}}$

$$
\text { (2) } \quad \mathrm{qe}=\frac{(C 0-C e q) V}{m}
$$

Donde $\mathrm{C}_{0}$ y $\mathrm{C}_{\mathrm{eq}}$ son las concentraciones iniciales y finales del ion metálico en la solución, V el volumen de la solución (L), m es la masa de la cáscara de plátano ( $\mathrm{g}$ ) y qe es la capacidad de adsorción de la biomasa (Abbasi, Z., Alikarami, M., Nezhad, E. R., Moradi, F., Moradi, V., 2013).

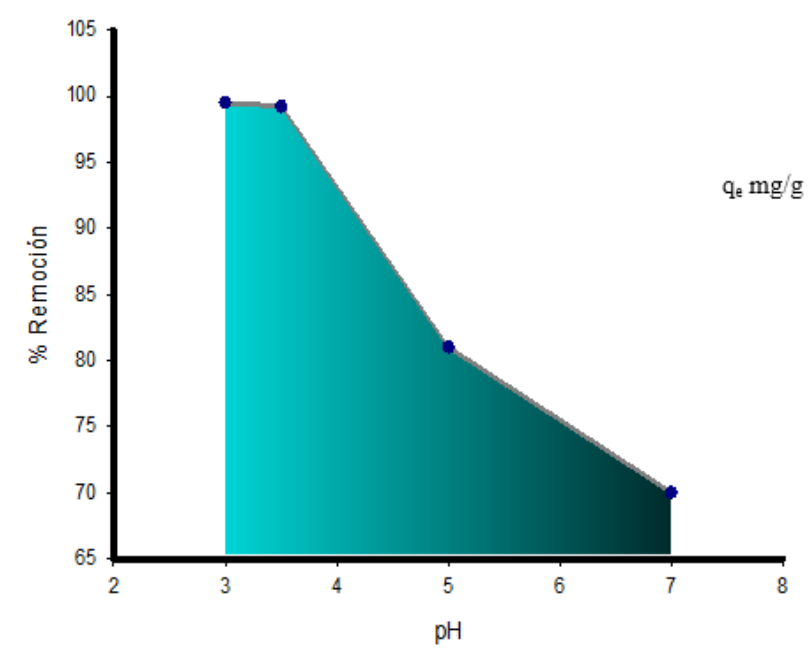

Figura 1. Efecto del pH en el \% de remoción

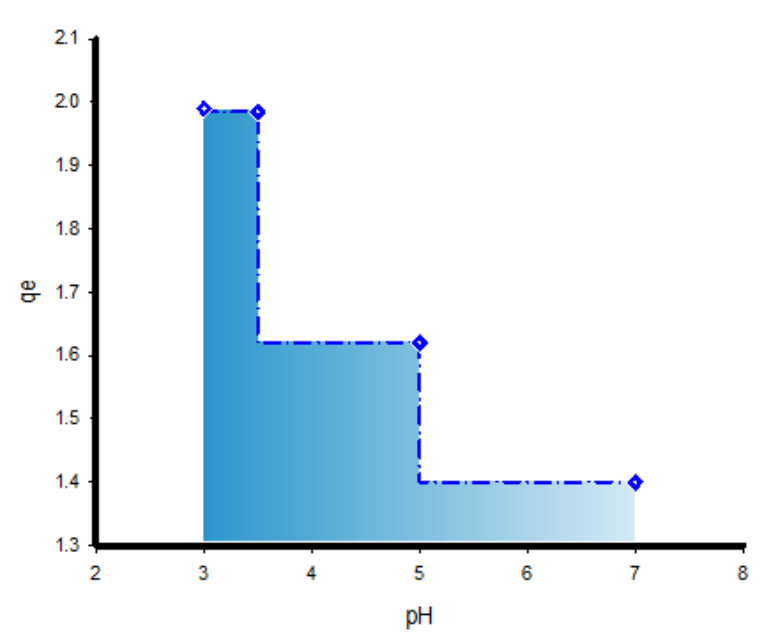

Figura 2. Efecto del pH en la capacidad de adsorción

En la figura 1, se observa que la remoción del metal es influenciada por el $\mathrm{pH}$, por lo que, el porcentaje de remoción disminuye hasta $70 \%$ al aumentar el $\mathrm{pH}$ a 7 . La figura 2, muestra que el pH también influyó en la capacidad de remoción de la cáscara de plátano, siendo éste inversamente proporcional. Ashraf A. et al. (2016), Memon J. R et al. (2009) y Kumar M. et al. (2014) obtuvieron resultados similares a este estudio al evaluar la remoción del cromo en 
función del pH. Figura 3. (Ashraf, A., Khalid S. b., Fazal, M., 2016) (Memon, J. R., Memon, S. Q., Bhanger, M. I., Khuhawar, M. Y., 2009) (Kumar,M., Majumder, C. B., 2014)

El Cromo hexavalente, se encuentra como $\mathrm{HCrO}_{4}^{-}, \mathrm{Cr}_{2} \mathrm{O}_{7}^{2-}, \mathrm{CrO}_{12}{ }^{-}, \mathrm{Cr}_{4} \mathrm{O}_{13^{2-}}, \mathrm{Cr}_{3} \mathrm{O}_{10^{2-}}$, una disminución en el $\mathrm{pH}$ provoca la protonación de la superficie del adsorbente, lo que induce una fuerte atracción por los iones de cromo de la solución cargados negativamente, por lo que la adsorción incrementa al aumentar la acidez de la solución. Sin embargo, al aumentar el pH se incrementa las concentraciones de los iones $\mathrm{OH}^{-}$induciendo cambios en la superficie del adsorbente, impidiendo la bioadsorción de los iones de cromo cargados negativamente, lo cual disminuye la adsorción del metal. (Torres, L. , Cárdenas, J. F., Moctezuma, M. G., Martínez, V. M., Acosta, I., 2012)

(a) En este estudio

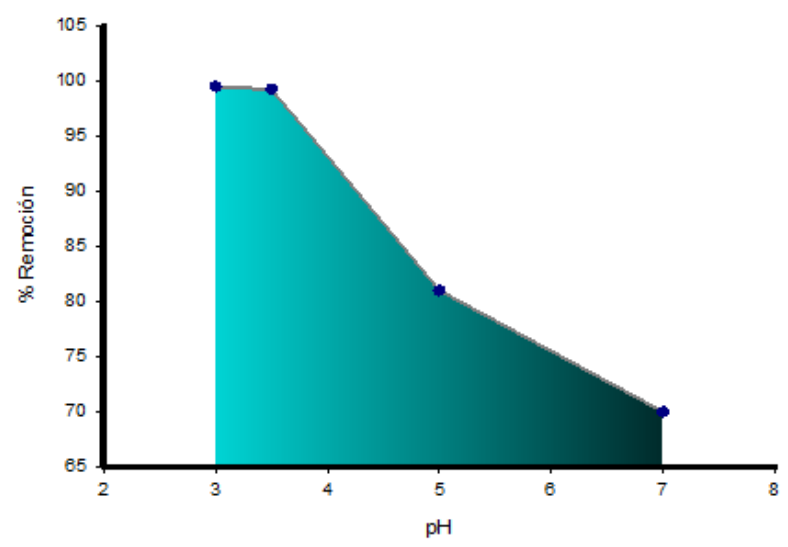

(C) Memon J. R et al.

Fuente: (Memon, J. R., Memon, S. Q., Bhanger, M.

I., Khuhawar, M. Y., 2009)

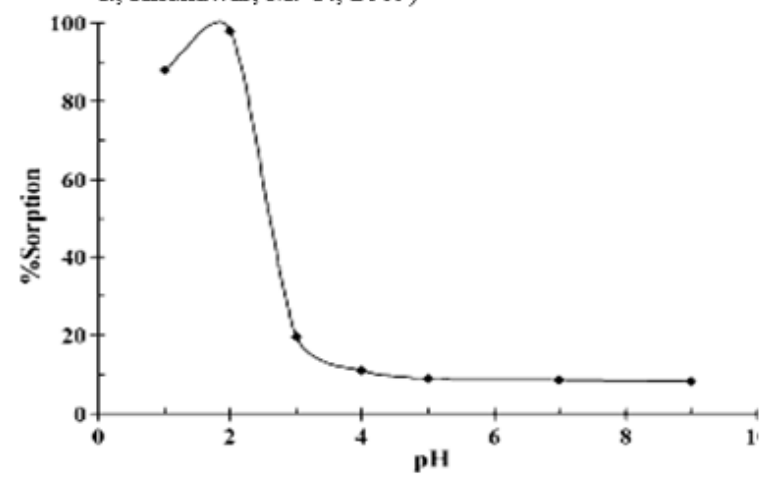

(b) Ashraf A. et al.

Fuente: (Ashraf, A., Khalid S. b., Fazal, M., 2016)

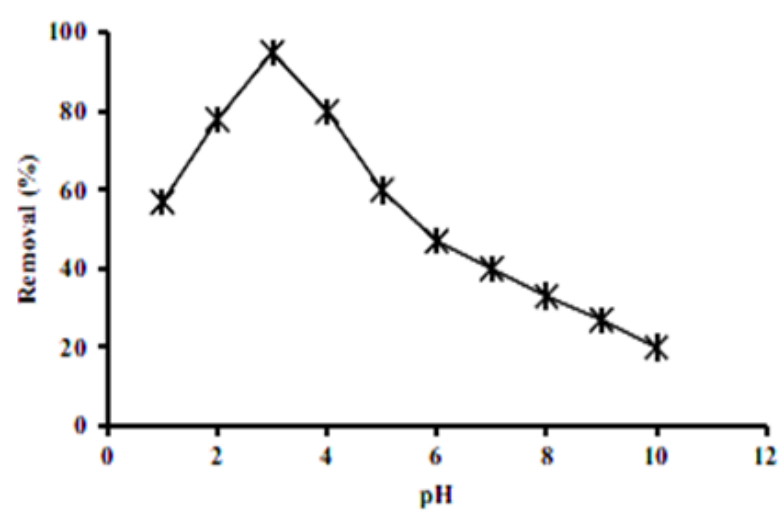

(d) Kumar M. et al.

Fuente: (Kumar,M., Majumder, C. B., 2014)

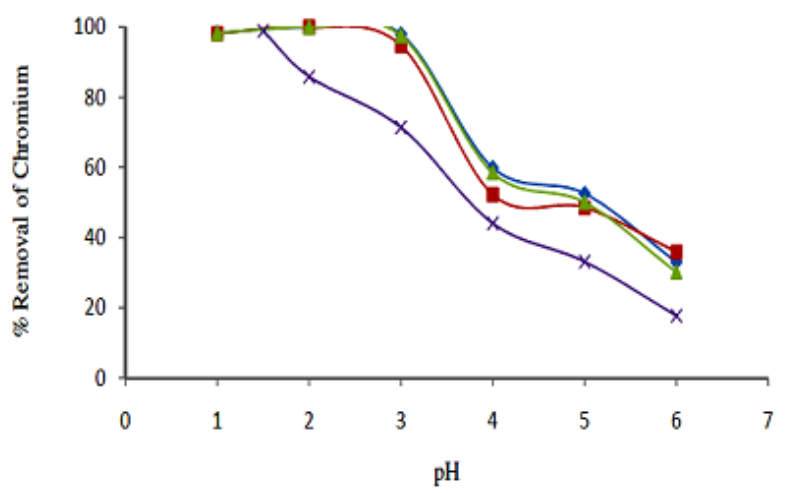

Figura 3. (a), (b), (c) y (d) Comparación de resultados obtenidos con otros estudios al evaluar el \% Remoción vs pH utilizando cáscara de plátano para remover Cr VI

En la figura 4, se observa que en el pH3, al aumentar la concentración de cromo

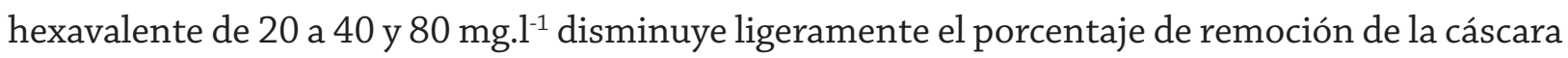
de plátano, no obstante, la figura 5 muestra que al aumentar la concentración incrementa la 
capacidad de adsorción de la cáscara de plátano. Este mismo comportamiento se ve reflejado para el pH 3,5 (Figura 6 y 7). Abbasi Z. et al. (2013) en su publicación menciona que "la capacidad de adsorción de la cáscara de plátano para $\mathrm{Co}^{2+} \mathrm{y} \mathrm{Ni}^{2+}$ incrementaba al aumentar la concentración de equilibrio". (Abbasi, Z. Alikarami, M., Nezhad, E. R., Moradi, F. Moradi, V., 2013)

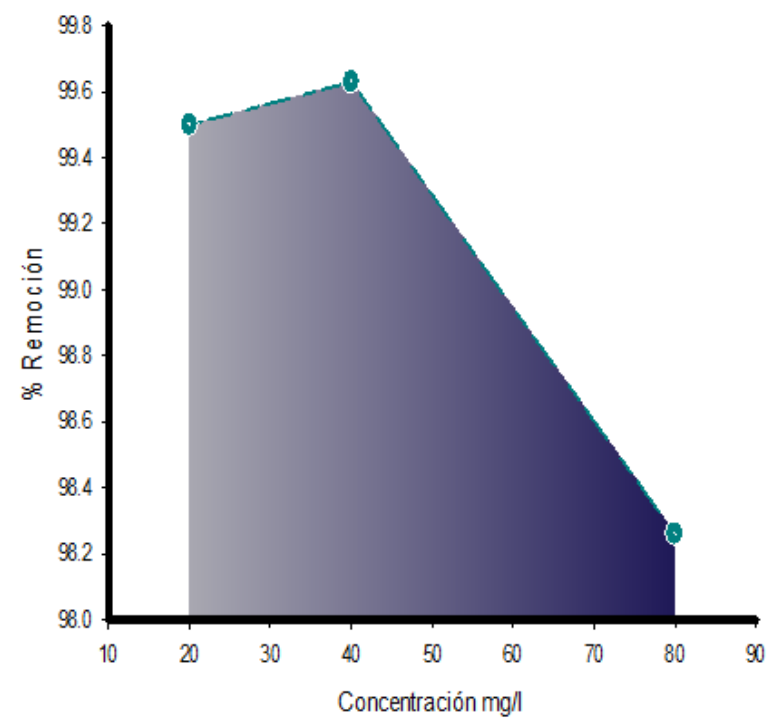

Figura 4. \% de Remoción versus concentración a pH3, segunda etapa

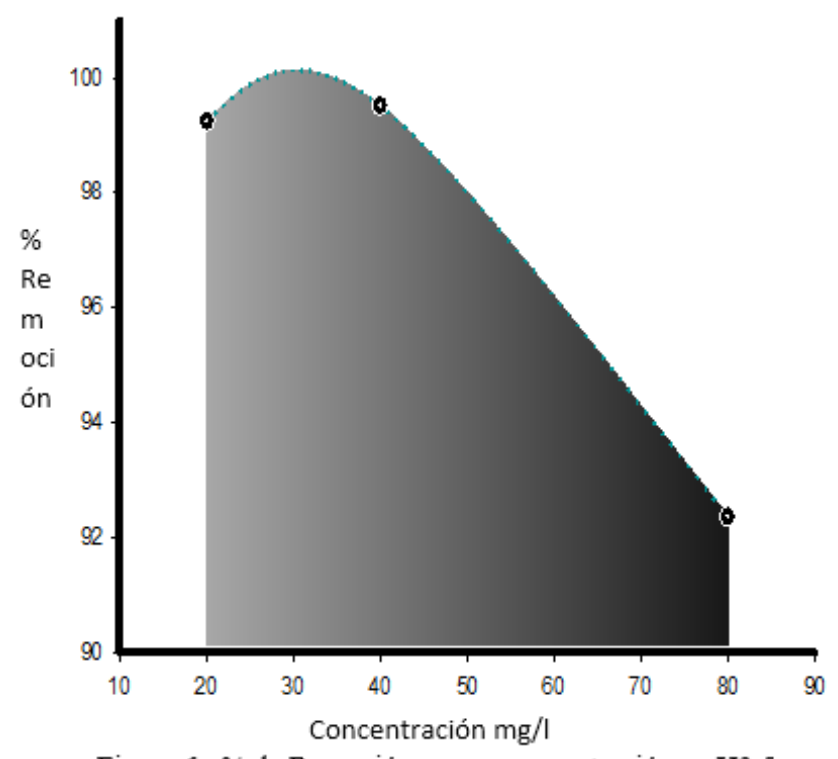

Figura 6. \% de Remoción versus concentración a pH3.5, segunda etapa

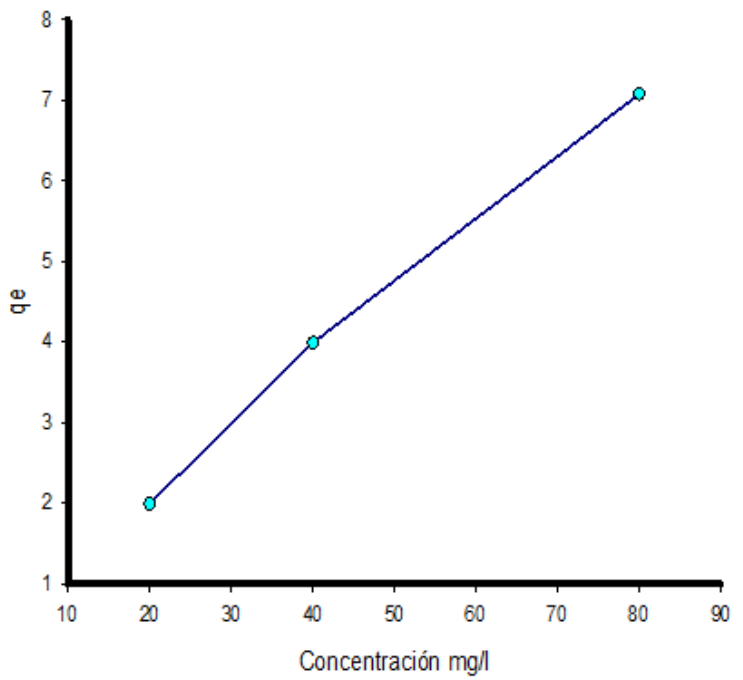

Figura 5. \% de Remoción versus concentración a $\mathrm{pH} 3$, segunda etapa

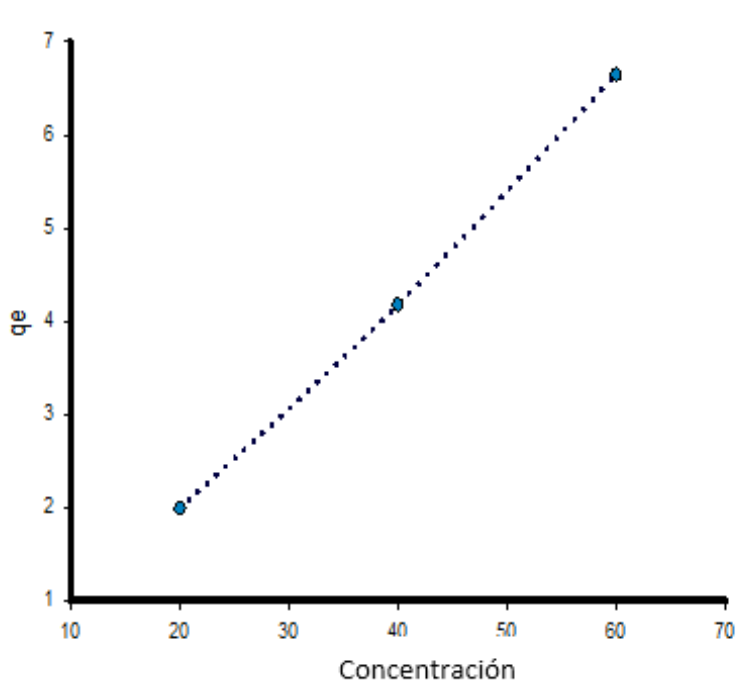

Figura 7. \% de Remoción versus concentración a pH3, 5 segunda etapa

Según los datos obtenidos, la cáscara de plátano fue capaz de remover más del 92\% de cromo hexavalente en la solución. Otro aspecto importante, es que la biomasa logró remover casi inmediatamente la mayor concentración del metal. 


\section{Isotermas de adsorción}

Las ecuaciones de Langmuir y Freundlich se usan comúnmente para describir el equilibrio de adsorción en aplicaciones de tratamiento de aguas contaminadas. Un aspecto fisicoquímico importante para la evaluación del proceso de adsorción es el equilibrio de isotermas. Las relaciones de equilibrio entre el adsorbente y el adsorbato se describen mediante las isotermas de adsorción. El modelo de isoterma de Freundlich supone que la adsorción ocurre en superficies heterogéneas (Abbasi, Z., Alikarami, M., Nezhad, E. R., Moradi, F., Moradi, V., 2013). La ecuación no lineal y linealizada de Freundlich se expresa como:

$$
\begin{gathered}
q e=a_{F} C_{e q}^{b F} \\
\log q e=\log a_{F}+b_{F} \cdot \log C_{e q}
\end{gathered}
$$

Donde $\boldsymbol{a} \boldsymbol{F}$ es la constante de la isoterma de Freundlich y $\boldsymbol{b}$ es el exponente de Freundlich que se obtienen de la pendiente y el intercepto al graficar $\operatorname{Logq}$ e vs $\log C_{\text {eq. }}$ (M. Romero-Sevilla, S. Sánchez-Cuadra, M. Benavente Silva, 2018). (Figura 8)

La isoterma de Langmuir supone que se forma una monocapa del metal sobre una superficie adsorbente relativamente regular, utilizando los grupos parcialmente protonados del adsorbente. (Abbasi, Z., Alikarami, M., Nezhad, E. R., Moradi, F., Moradi, V., 2013) La ecuación no lineal y linealizada de Langmuir se expresa como:

$$
\frac{\mathrm{Ceq}}{\mathrm{qe}}=\frac{1}{q e \max } \mathrm{Ceq}+\frac{1}{q e \max b}
$$

Donde qemax es la máxima capacidad de adsorción $(\mathrm{mg} / \mathrm{g}$ ) y b es la constante de equilibrio de adsorción de Langmuir, estas dos constantes se obtuvieron del intercepto y la pendiente

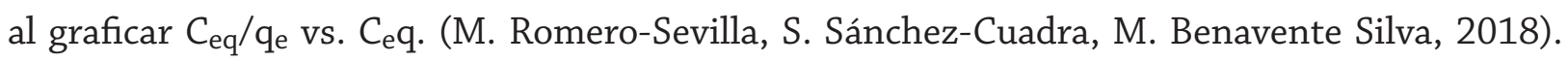
(Figura 9) 
(a)

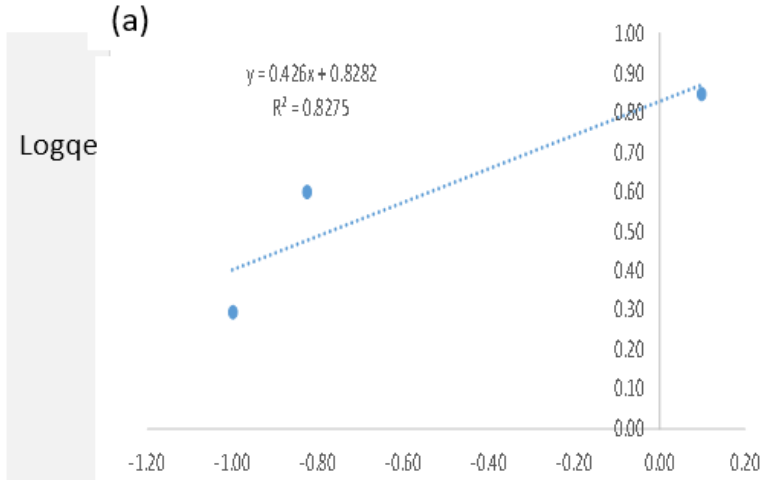

(b)

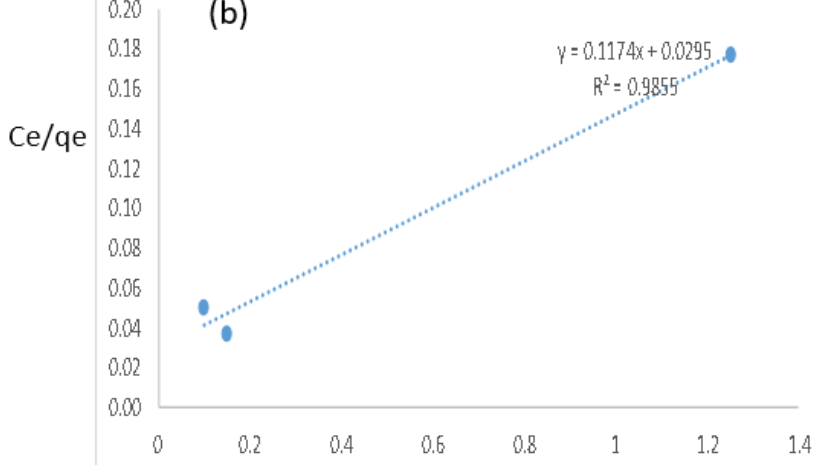

Figura 8. a y b Ecuación linealizada de isotermas de adsorción de Freundlich y Langmuir pH3

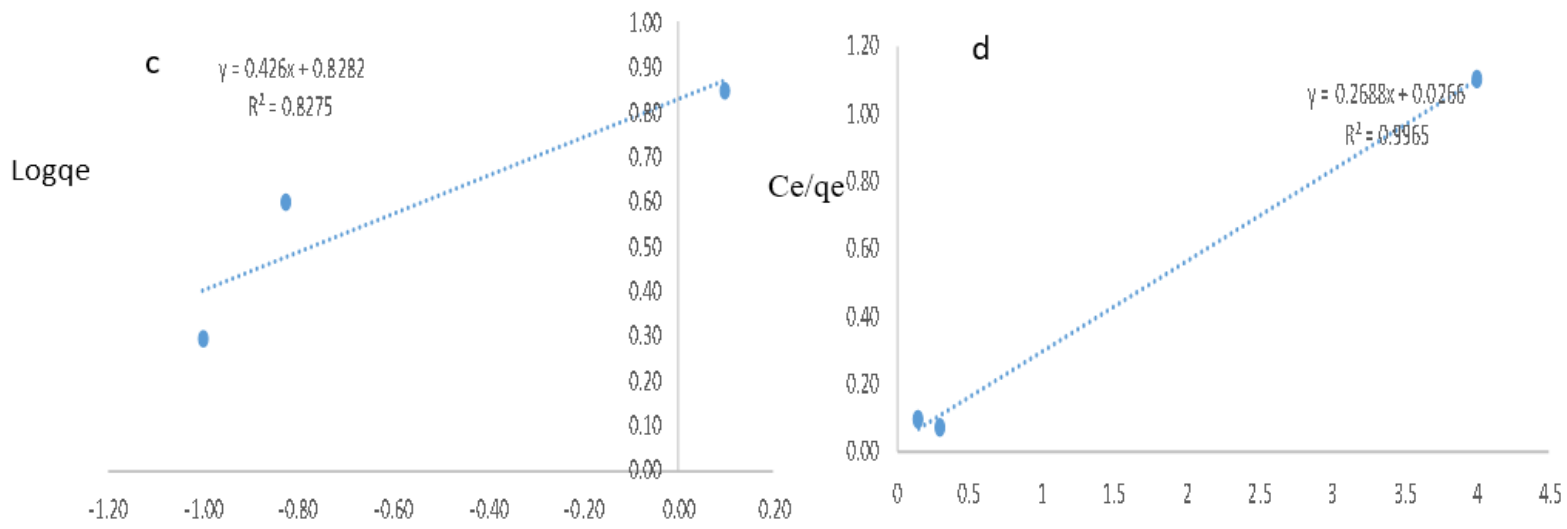

Figura 9. c y d Ecuación linealizada de isotermas de adsorción de Freundlich y Langmuir pH3.5

Las Figuras 8 y 9, muestran las ecuaciones linealizadas de ambos modelos, de los cuales el mejor modelo de equilibrio se determinó en base al coeficiente de correlación $\left(\mathrm{R}^{2}\right)$. El equilibrio de adsorción de metal se observa mejor representado por la isoterma de Langmuir, que supone la existencia de adsorción en monocapa en una superficie homogénea. La mayor capacidad de adsorción se obtuvo para $\mathrm{pH} 3$, lo que indica que el $\mathrm{pH}$ influye en la capacidad de adsorción del metal. Los resultados corresponden a lo descrito por Abbasi Z. et al. (2013) en su publicación, donde el mejor modelo de isoterma para la remoción de $\mathrm{Co}^{2+} \mathrm{y} \mathrm{Ni}^{2+}$ utilizando cáscara de plátano se ajusta a Lamguir. (Abbasi, Z. Alikarami, M., Nezhad, E. R., Moradi, F. Moradi, V., 2013) En la tabla 1 se muestran los valores de $R^{2}$ y capacidad máxima de adsorción de la cáscara plátano para ambos modelos. 
Tabla 1. Constantes de isotermas de adsorción de Freundlich y Langmuir

\begin{tabular}{|c|c|c|c|c|c|c|}
\hline & \multicolumn{3}{|c|}{ Isoterma Freundlich } & \multicolumn{3}{c|}{ Isoterma Langmuir } \\
\hline $\mathbf{P H}$ & $\mathbf{a f} \mathbf{~ m g / g}$ & $\mathbf{b}$ & $\mathbf{R}^{\mathbf{2}}$ & $\mathbf{q} \mathbf{m a x} \mathbf{~ m g / g}$ & $\mathbf{b}$ & $\mathbf{R}^{\mathbf{2}}$ \\
\hline $\mathbf{3 . 0}$ & 6.894 & 0.426 & 0.8275 & 8.5179 & 0.0118 & 0.9855 \\
\hline $\mathbf{3 . 5}$ & 4.723 & 0.324 & 0.8184 & 3.7202 & 0.0072 & 0.9965 \\
\hline
\end{tabular}

La isoterma de Langmuir se puede expresar en términos de una constante adimensional llamada factor de separación ( $\mathrm{R}_{\mathrm{L}}$, también llamado parámetro de equilibrio) que se define mediante la siguiente ecuación:

$$
\mathrm{R}_{\mathrm{L}}=1 / 1+\mathrm{bCo}
$$

Donde $\mathrm{C}_{0}(\mathrm{mg} / \mathrm{L})$ es la concentración inicial del cromo hexavalente y $\boldsymbol{b}$ es la constante de Langmuir relacionada con la energía de adsorción. El valor de $\mathrm{R}_{\mathrm{L}}$ indica que la forma de las isotermas es: desfavorable $\left(R_{L}>1\right)$, lineal $\left(R_{L}=1\right)$, favorable $\left(0<R_{L}<1\right)$ o irreversible $\left(R_{L}=0\right)$. (B. Meroufel, O. Benali, M. Benyahia , 2013). Figura 9 y 10

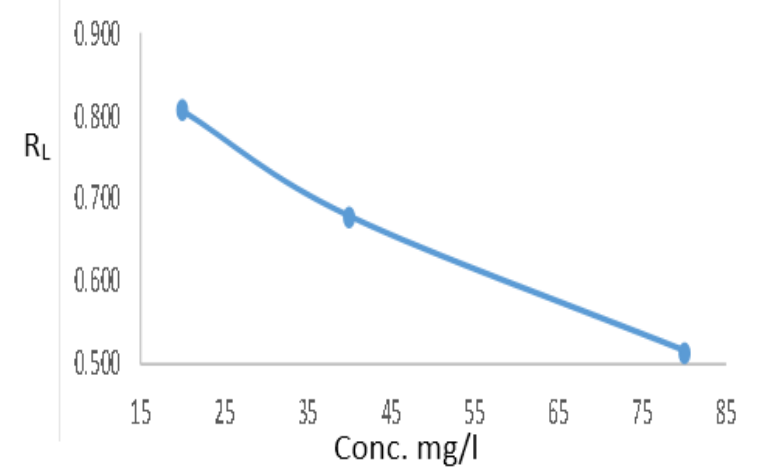

Figura 10. Factor RL de la cáscara de plátano para pH3

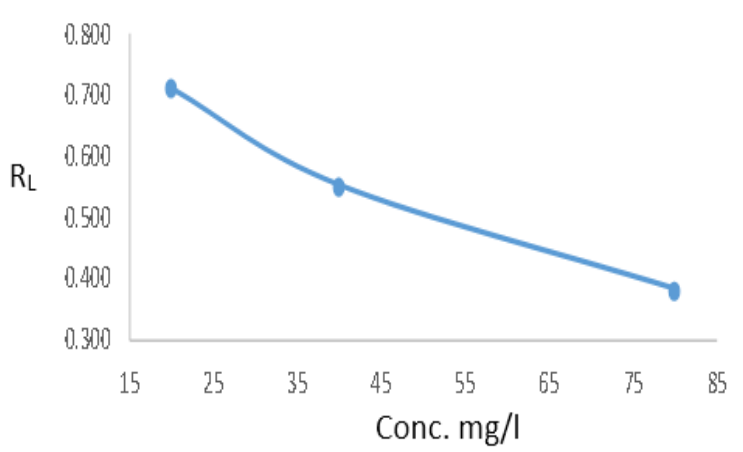

Figura 11. Factor RL de la cáscara de plátano para pH3.5

Según las figuras 10 y 11 , los valores de $R_{L}$ son más bajos a concentraciones $\left(C_{0}\right)$ más altas de Cromo hexavalente, es decir que, a mayor concentración, más bajo es el factor de separación $\left(\mathrm{R}_{\mathrm{L}}\right)$, este comportamiento es similar en ambos $\mathrm{pH}$. Los valores de $\mathrm{R}_{\mathrm{L}}$ obtenidos oscilan entre 0,809 a 0,383, indicando una adsorción favorable de cromo hexavalente en la cáscara de plátano. Los resultados corresponden a lo descritos por Meroufel B.et al. (2013) en su publicación donde el factor $\mathrm{R}_{\mathrm{L}}$ tendió a cero a medida que aumentó la concentración. (B. Meroufel, O. Benali, M. Benyahia , 2013). Además, Deshmukh P. D. et al. (2017), en su investigación obtuvo una adsorción favorable de cadmio en la cáscara de plátano con un factor de separación menor a 1. (Deshmukh, P. D., Khadse, G.K., Shinde, V. M., Labhasetwar, P., 2017) De igual manera, Memon J. R et al. (2008) en su publicación, menciona que obtuvo valores $R_{L}<1$, lo que muestra una adsorción favorable de cromo trivalente en la cáscara de plátano. (Memon, J. R., Memon, S. Q., Bhanger, 
M. I., Khuhawar, M. Y., 2009). En base a lo anterior, la cáscara de plátano tiene una capacidad de adsorción favorable para la remoción de cromo hexavalente en aguas contaminadas.

\section{CONCLUSIONES}

El mayor porcentaje de remoción de Cromo hexavalente se obtuvo a pH 3 y 3,5 alcanzando un porcentaje de remoción mayor a 92.

La capacidad de adsorción de la cáscara de plátano incrementó a medida que aumentó la concentración del metal, siendo ambos directamente proporcional.

El equilibrio de adsorción a pH3 y 3,5 fue mejor representada por la isoterma de Langmuir que la de freundlich, obteniendo mayor capacidad de adsorción máxima (qemax) en el pH3.

El factor de separación fue menor a $1\left(R_{L}<1\right)$, lo que indica una adsorción favorable del cromo hexavalente en la cáscara de plátano.

La cáscara de plátano es un excelente material para remover el contenido de cromo hexavalente en aguas contaminadas, lo cual podría ser utilizada para el tratamiento de aguas residuales contaminadas por este metal pesado.

\section{Agradecimientos}

A la Vice-Rectoría de Investigación, Posgrado y Extensión Universitaria, y a la Dirección de Investigación de la UNAN-Managua por el financiamiento a través de los Fondos para Proyectos de Investigación (FPI).

Al Comedor Central de la Universidad Nacional Autónoma de Nicaragua, Managua (UNAN-Managua) y al Comedor del Centro para Investigación de Recursos Acuáticos (CIRAUNAN) por proporcionar las cáscaras de plátanos necesarias para la investigación.

Al Lic. Jorge Esquivel Quezada por su valiosa colaboración técnica durante el proceso de investigación.

\section{REFERENCIAS BIBLIOGRÁFICAS}

Al-Azzawi, M.N.A., Shartooh, S.M., Al-Hiyaly S.A.K. (2013). The Removal of Zinc,
BIOSORPTION MECHANISM. International Journal of Science Technology \& Management, 1-7.

Abbasi, Z. Alikarami, M., Nezhad, E. R., Moradi, F. Moradi, V. (2013). Adsorptive Removal of $\mathrm{Co} 2+$ and $\mathrm{Ni2}+$ by Peels of Banana from Aqueous Solution. Universal Journal of Chemistry, 90-95. 
APHA, AWWA, WEF. (2012). Standard Methods for the Examination of Water and Wastewater. 22nd ed. En 3500-Cr B. Colorimetric Method (págs. 69-70). Washington: American Public Health Association.

Asamblea Nacional. (30 de 11 de 2017). DECRETO EJECUTIVO Nº 21-2017. LA GACETA- DIARIO OFICIAL, pág. 48.

Ashraf, A., Khalid S. b., Fazal, M. (2016). Removal of chromium (VI) from aqueous medium using chemically modified banana peels as efficient low-cost adsorbent. Elsevier, 1-10.

B. Meroufel, O. Benali, M. Benyahia . (2013). Adsorptive removal of anionic dye from aqueous solutions by Algeriankaolin: Characteristics, isotherm, kinetic and thermodynamic studies. J. Mater. Environ. Sci., 482-491.

Deshmukh, P. D., Khadse, G.K., Shinde, V. M., Labhasetwar, P. (2017). Cadmium Removal from Aqueous Solutions Using Dried Banana Peels as An Adsorbent: Kinetics and Equilibrium Modeling. Journal of Bioremediation \& Biodegradation, 8: 395 . doi: 10.4172/2155-6199.1000395.

Díaz, M. R., Contreras, R., Guardiola, M. A., Mayo del Río, C. (2016). Kinetic study of absorption of chromium (VI) using Canary Bananas Peels in contaminated water. International Journal of Innovation and Scientific Research, 139145.
EPA. (January de 2000). Chromium Compounds. Obtenido de https://www. epa.gov/sites/production/files/2016-09/ documents/chromium-compounds.pdf

Guertin J. (2004). Toxicity and Health Effects of Chromium (All Oxidation States). Chromium (VI) Handbook, 215-234.

Kumar,M., Majumder, C. B. (2014). Bioabsorptive Removal of $\mathrm{Cr}$ (VI) from Stimulated Tannery Waste Water using Different Adsorbents and Compare with GAC. Krishi Sanskriti, 73-86.

M. Romero-Sevilla, S. Sánchez-Cuadra, M. Benavente Silva. (2018). APLICACIÓN DE QUITOSANO MODIFICADO EN EL TRATAMIENTODEAGUAS RESIDUALES DE TENERÍAS. Nexo Revista Científica, 104-119.

Memon, J. R., Memon, S. Q., Bhanger, M. I., Khuhawar, M. Y. (2009). Banana Peel: A Green and Economical Sorbent for $\mathrm{Cr}$ (III) Removal. Pak. J. Anal. Environ. Chem, 20-25.

OSHA . (2009). Hexavalent Chromium. Washington.

Torres, L. , Cárdenas, J. F., Moctezuma, M. G., Martínez, V. M., Acosta, I. (2012). Remoción de cromo hexavante por la cáscara de plántano (Musa cavendishii). QUÍMICA HOY CHEMESTRY SCIENCES, 29-32. 